\title{
TSUNAMI LEAD WAVE RECONSTRUCTION BASED ON NOISY SEA SURFACE HEIGHT MEASUREMENTS
}

\author{
Kegen $\mathrm{Yu}$ \\ School of Geodesy and Geomatics and Collaborative Innovation Center for Geospatial Technology, Wuhan University, \\ Wuhan 430079, China - kgyu@sgg.whu.edu.cn
}

KEY WORDS: Tsunami lead wave reconstruction, Wavelet theory, Sea surface height measurement, GNSS reflectometry

\begin{abstract}
:
This paper presents a Tsunami lead wave reconstruction method using noisy sea surface height (SSH) measurements such as observed by a satellite-carried GNSS reflectometry (GNSS-R) sensor. It is proposed to utilize wavelet theory to mitigate the strong noise in the GNSS-R based SSH measurements. Through extracting the noise components by high-pass filters at decomposition stage and shrinking the noise by thresholding prior to reconstruction, the noise is greatly reduced. Real Tsunami data based simulation results demonstrate that in presence of SSH measurement error of standard deviation $50 \mathrm{~cm}$ the accuracy in terms of root mean square error (RMSE) of the lead wave height (true value $145.5 \mathrm{~cm}$ ) and wavelength (true value $592.0 \mathrm{~km}$ ) estimation is 21.5 $\mathrm{cm}$ and $56.2 \mathrm{~km}$, respectively. The results also show that the proposed wavelet based method considerably outperforms the Kalman filter based method on average. The results demonstrate that the proposed wave reconstruction approach has the potential for Tsunami detection and parameter estimation to assist in achieving reliable Tsunami warning.
\end{abstract}

\section{INTRODUCTION}

A Tsunami is a specific ocean wave usually created by a natural process such as earthquake, volcano eruption, or landslide. Depending on how a Tsunami is triggered, The 2004 Indian Ocean Tsunami and the 2011 Japan's Tsunami were both triggered by strong earthquakes. These two Tsunamis were so powerful and disastrous, causing huge damage and many life losses. That is why Tsunami warning is vital to reduce economic and life losses dramatically in the event of a strong Tsunami. A number of Tsunami warning systems or networks have been established on the globe (Bernard and Meinig, 2011; Falck et al, 2010) These systems basically make use of measurements from a network of seismic sensors installed on the ground or on the bottom of the ocean to derive the Tsunami parameters so as to deliver warning information. Real time Tsunami monitoring may be realized by using measurements from waverider buoys equipped with sea surface height (SSH) measurement sensors. Although such a network of buoys only covers a rather limited part of the ocean, they can provide accurate information of some Tsunamis which happen to propagate through the buoys. Satellite altimetry is another technique for real time Tsunami monitoring, providing SSH measurements with accuracy of a few centimeters (NOAA, 2016). However, due to the limited coverage of a satellite altimeter, the chance that a Tsunami is captured by an altimetry satellite is rather small. That is why only a few out of more than one hundred Tsunami events were successfully identified by a satellite altimeter since the launching of the Topex satellite by NASA in 1993 (Hamlington, 2010).

The main theme of the paper is the reconstruction of Tsunamis by use of GNSS-R based SSH measurements. In particular, we propose to exploit wavelet theory to mitigate measurement noise effectively. It is a fact that there is a wide range of filtering or smoothing methods which can be utilized for noise suppression (Anderson and Moore, 2005). However, wavelet based noise mitigation has been widely investigated in signal and image processing and is particularly suited for nonstationary time-limited or transient signals (Daubechies, 1992; Mallat, 2008). Through wave reconstruction, Tsunami pattern and characteristics can be better identified. It is assumed that Tsunami detection is first performed by using GNSS-R techniques such as the methods proposed in (Stosius et al, 2010; $\mathrm{Yu}, 2016)$. Once a Tsunami is detected, Tsunami reconstruction is carried out to recover the original wave pattern which may be exploited to estimate Tsunami parameters (propagation direction and speed) (Yu, 2015).

The remainder of the paper is organized as follows. Section 2 describes the procedure to use wavelet theory to enable lead wave reconstruction. Section 3 shows the simulation results using real Tsunami lead wave data, and Section 5 gives the concluding remarks.

\section{WAVELET BASED RECONSTRUCTION METHOD}

The proposed lead wave reconstruction makes use of the wavelet theory to process a sequence of SSH measurement data samples such as produced with GNSS-R technique. It is assumed that the presence of a Tsunami lead wave is detected so that the sequence of data samples includes the lead wave samples. The functional diagram of the proposed wavelet based method is shown in Figure 1 (on the bottom of next page).

Signal decomposition is first carried out by selecting the wavelet and the number of levels, which is constrained by the number of samples. The sequence of SSH measurement samples are simultaneously high-pass and low-pass filtered and then down-sampled. The low-pass filtered and down-sampled data sequence is again high-pass and low-pass filtered and then down-sampled. The procedure repeats for the predefined number of levels. The down-sampled output from each high- 
pass filter is then processed to mitigate the noise through thresholding. Signal reconstruction is then performed to reconstruct the lead wave using the high-pass and low-pass filters, up-sampling, the data sequence from the low-pass filter of the final stage (or level), and the data sequences resulting from processing data sequences generated by the high-pass filters during signal decomposition and noise mitigation. The reconstructed lead wave is then modeled mathematically using triangular functions (Yu, 2016) so that the wave heights and wave lengths can readily be determined.

\section{REAL SATELLITE TSUNAMI DATA BASED SIMULATION RESULTS}

\subsection{Tsunami Lead Waves Observed by Altimetry Satellites}

When a Tsunami is observed by a satellite-carried sensor, the observed lead wave shape can be quite different from that observed by a buoy-based sensor, depending on the satellite moving direction and the SSH measurement accuracy. For instance, if the satellite is moving exactly in the same (or opposite to the) direction of the Tsunami propagation, the length of the observed wave is slightly longer (or shorter) than that of the real one. In some cases, the observed wave length by a satellite sensor can be significantly longer.

Figure 2 shows the two lead waves of the Tsunami (triggered by 2011 Japan's earthquake) observed by Jason-1 altimetry satellite (pass 146, cycle 338) and Envisat satellite (pass 449, cycle 100). The distance is calculated using the sampling time intervals and the satellite ground velocity which is about 5.8 $\mathrm{km} / \mathrm{s}$ for Jason-1 and $6.86 \mathrm{~km} / \mathrm{s}$ for Envisat. The start distance point on the horizontal axis is arbitrary. There are a few sampling time intervals in which SSH measurements are not available so that they are simply replaced with zeros. The data were downloaded from (Tudelft, 2016) which is managed by the Technical University of Delft, the Netherlands.

The initially sensed SSH data were affected by a range of factors including troposphere, ionosphere, barometer, dynamic atmosphere, solid earth tide, ocean tide, load tide, pole tide and sea state bias. The downloaded data are the processed data after those effects are corrected. Thus, the resultant SSH (or sea level anomaly) could only consist of components contributed by the Tsunami, the residual error from the various corrections, and the SSH measurement error. The measurement accuracy of the Envisat and Jason-1 altimeters is about a few centimeters, while the residual error might also be a number of centimeters due to the increasingly enhanced performance in the modeling of the various factors. The sea surface locations of the two lead waves are listed in Table 1.
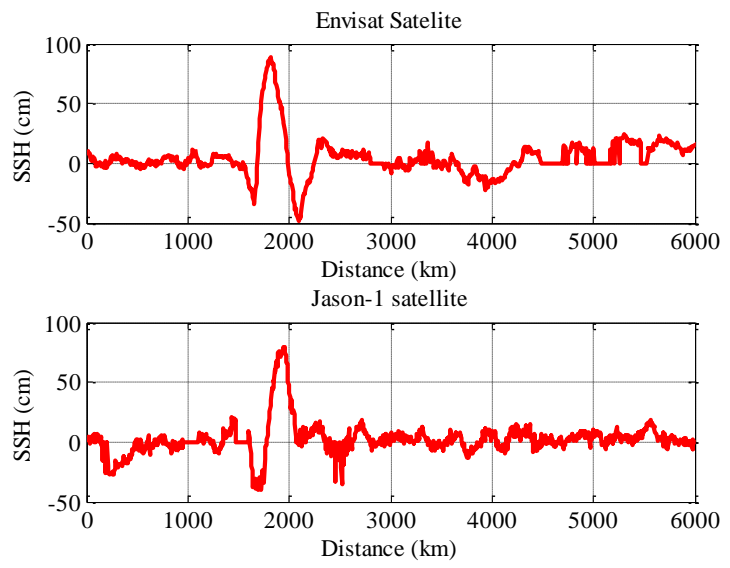

Figure 2, Tsunami lead waves triggered by 2011 Japan's earthquake and observed by Envisat and Jason-1 altimetry satellites.

Table 1. Locations of two Tsunami lead waves observed by two altimetry satellites.

\begin{tabular}{|c|c|c|c|c|}
\hline \multirow{2}{*}{ Satellite } & \multicolumn{2}{|c|}{ Latitude (deg) } & \multicolumn{2}{c|}{ Longitude (deg) } \\
\cline { 2 - 5 } & Start point & End point & Start point & End point \\
\hline Jason-1 & -38.2351 & -41.9297 & 40.4309 & 43.0984 \\
\hline Envisat & -36.4802 & -30.3041 & 156.4616 & 154.6962 \\
\hline
\end{tabular}

\subsection{Simulation Results}

In this section modeled GNSS-R based SSH measurements using real Tsunami lead wave data observed by satellite Envisat

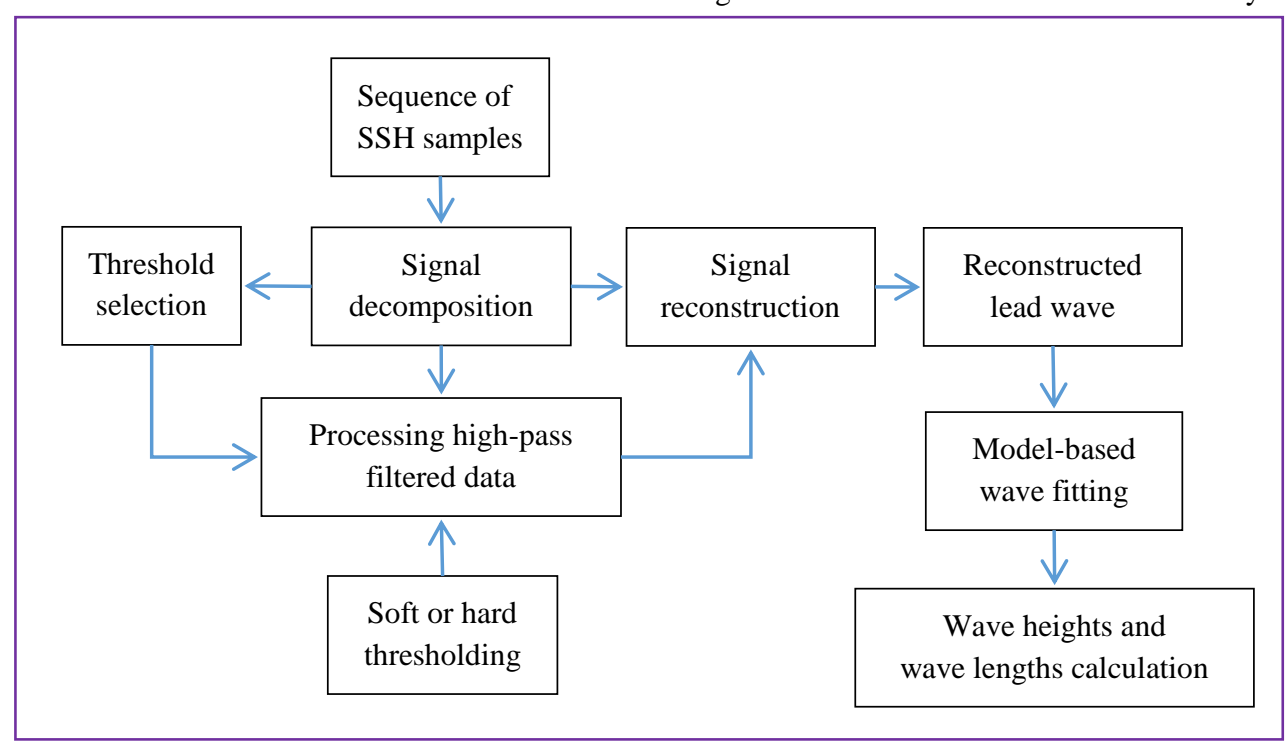

Figure 1. Block diagram of the wavelet based Tsunami lead wave reconstruction approach 
as shown in the upper panel of Figure 2 are used to evaluate the proposed Tsunami lead wave reconstruction approach. The SSH measurements are corrupted by Gaussian noise with a zero mean and standard deviation of $50 \mathrm{~cm}$ as shown in Figure 3. The distance interval is $6.86 \mathrm{~km}$ equivalent to sampling period or incoherent integration time of one second.

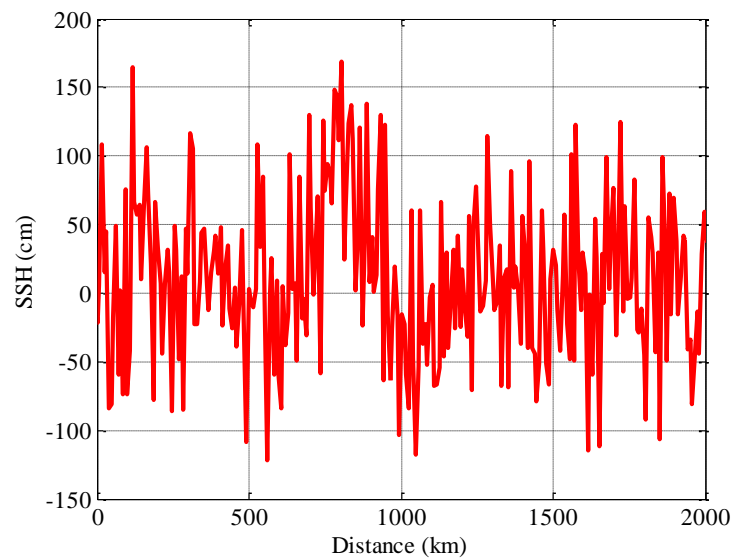

Figure 3. Modeled SSH measurements by adding noise of STD of $50 \mathrm{~cm}$ to Envisat SSH data.

A number of wavelets including Daubechies, Meyer, Coiflet, and Biorhtogonal wavelets are widely used in signal and image processing. When wavelet parameters are properly chosen, the mentioned four wavelets produce very similar reconstruction results. Figure 4 shows the impact of noise standard deviation on the reconstruction performance when four different standard deviation values are evaluated and the Daubechies wavelet is used. As expected, the performance degrades as the noise standard deviation increases. However, the impact is mainly on the lead wave height, while the reconstructed wavelength is only slightly affected. When noise standard deviation is $90 \mathrm{~cm}$, useful information about the lead wave especially the height and length can still be obtained.
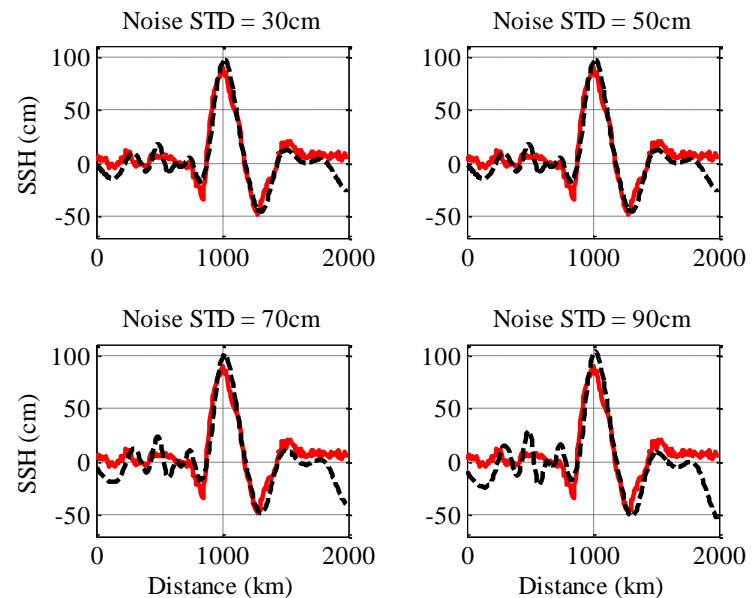

Figure 4. Effect of measurement noise standard deviation on lead wave reconstruction.

Kalman filter (KF) has been widely employed to reduce noise and smooth data (Grewal and Andrews, 2008). Therefore, it is useful to evaluate the performance of the KF-based lead wave reconstruction. Figure 5 shows the filtering results when setting the noise standard deviation to be $50 \mathrm{~cm}$ and using a linear $\mathrm{KF}$ with state variance selected as $0.1 \mathrm{~cm}^{2}$ and observation variance selected as $2500 \mathrm{~cm}^{2}$. By comparing with the modeled SSH measurements shown in Fig. 3, one can see that the noise is significantly reduced. However, the performance of the KF-based reconstruction is considerably inferior when compared to the wavelet based reconstruction approach in terms of wave heights. The reconstructed lead wave is also shifted slightly, although this may not be a significant issue.

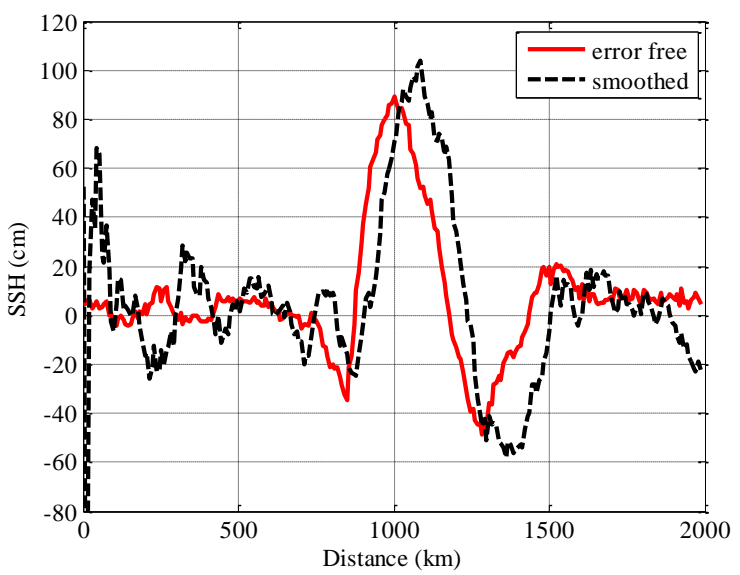

Figure 5. Linear Kalman filter based Tsunami lead wave reconstruction results.

The above results are obtained when giving a specific sequence of measurement noise samples. To have a better evaluation of the wavelet based reconstruction method, it is necessary to use a large number of noise sample sequences and use curve fitting to obtain the estimate of the wave heights and lengths from each sequence of data. Then the statistics of the lead wave parameter estimation errors can be determined. To achieve the goal, 200 sequences of noise samples are generated and a lead wave model is obtained from the reconstructed data associated with each sequence of the noise samples. The fitted wave height is $145.5 \mathrm{~cm}$ and the fitted wavelength is $592.0 \mathrm{~km}$. The resulting root mean square error (RMSE) of the wave height and wavelength estimation is $21.5 \mathrm{~cm}$ and $56.2 \mathrm{~km}$ respectively for the wavelet-based approach, while they are $31.5 \mathrm{~cm}$ and $65.0 \mathrm{~km}$ respectively for the KF based approach.

\section{CONCLUSIONS}

In this paper, wavelet theory has been exploited to mitigate $\mathrm{SSH}$ measurement noise for Tsunami lead wave reconstruction and wave parameter estimation. The performance of the proposed method was evaluated through extensive simulations using real Tsunami lead wave data and modelled SSH measurement noise samples. The results have demonstrated that accurate lead wave reconstruction and wave parameter estimation are possible when the lead wave height is significantly greater than the noise standard deviation.

Future work will focus on the evaluation of the proposed Tsunami reconstruction method using real Tsunami data observed by satellite-carried GNSS receivers. It is also useful to develop techniques to select the appropriate wavelet parameters to enable the good reconstruction performance. 


\section{ACKNOWLEDGEMENTS}

This work was supported by the National Natural Science Foundation of China under Grant 41574031.

\section{REFERENCES}

Anderson, B.D.O., and J.B. Moore, 2005. Optimal Filtering, Dover Publications.

Bernard, E.N., and C. Meinig, 2011. History and future of deep-ocean tsunami measurements. Proceedings of OCEANS, Kona, Hawaii, USA, pp. 1-7.

Daubechies, I., 1992. Ten Lectures on Wavelets, SIAM: Society for Industrial and Applied Mathematics, $1^{\text {st }}$ edition.

Falck, C., M. Ramatschi, M. Bartsch, and A. Merx, 2010. The GNSS-based component of the German-Indonesian Tsunami Early Warning System (GITEWS): Overview, first operation results and current developments. Proceedings of International Geoscience and Remote Sensing Symposium, Honolulu, HI, USA, pp. 134-137.

Grewal, M.S., and A.P. Andrews, 2008. Kalman Filtering: Theory and Practice Using Matlab. Wiley-IEEE Press, $3^{\text {rd }}$ edition.

Hamlington, B.D., R.R. Leben, O.A. Godin, and V.G. Irisov, 2010. On the feasibility of tsunami detection using satellitebased sea surface roughness measurements. Proceedings of International Geoscience and Remote Sensing Symposium, Honolulu, HI, USA, pp. 3035-3038.

Mallat, S., 2008. A Wavelet Tour of Signal Processing, Academic Press, $3^{\text {rd }}$ edition.

NOAA, 2016. http://nctr.pmel.noaa.gov/indo_1204.html

Stosius, R., G. Beyerle, M. Semmling, A. Helm, A. Hoechner, J. Wickert, and J. Lauterjung, 2010. Tsunami detection from space using GNSS reflections: results and activities from GFZ, Proceedings of International Geoscience and Remote Sensing Symposium, Honolulu, HI, USA, pp. 3047-3050.

Tudelft, 2016. http://rads.tudelft.nl/rads/rads.shtml

Yu, K., 2015. Tsunami-wave parameter estimation using GSSbased sea surface height measurement. IEEE Transactions on Geoscience and Remote Sensing, 53(5), pp. 2603-2611.

Yu, K., 2016. Weak Tsunami detection using GNSS-R based sea surface height measurement, IEEE Transactions on Geoscience and Remote Sensing, 54(3), pp. 1363-1375. 